\title{
Front Matter: Volume 7855
}

, "Front Matter: Volume 7855," Proc. SPIE 7855, Optical Metrology and Inspection for Industrial Applications, 785501 (8 December 2010); doi: 10.1117/12.886292

SPIE. Event: Photonics Asia 2010, 2010, Beijing, China 


\title{
PROCEEDINGS OF SPIE
}

\section{Optical Metrology and Inspection for Industrial Applications}

\author{
Kevin Harding \\ Peisen S. Huang \\ Toru Yoshizawa \\ Editors
}

\section{8-20 October 2010 \\ Beijing, China}

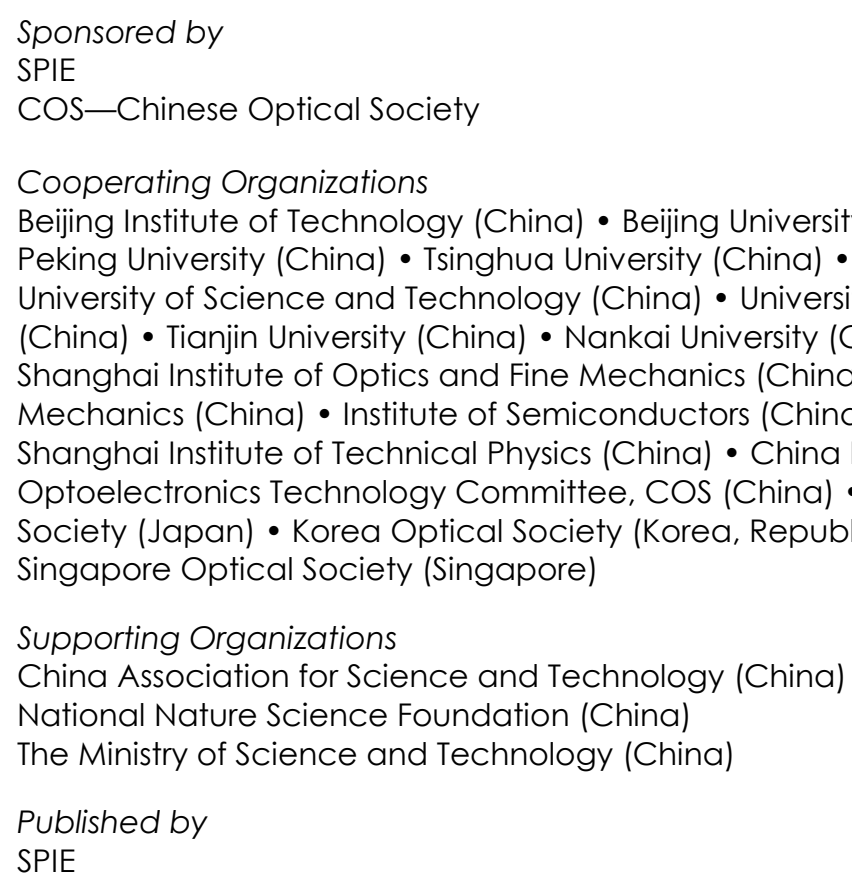

Volume 7855 
The papers included in this volume were part of the technical conference cited on the cover and title page. Papers were selected and subject to review by the editors and conference program committee. Some conference presentations may not be available for publication. The papers published in these proceedings reflect the work and thoughts of the authors and are published herein as submitted. The publisher is not responsible for the validity of the information or for any outcomes resulting from reliance thereon.

Please use the following format to cite material from this book:

Author(s), "Title of Paper," in Optical Metrology and Inspection for Industrial Applications, edited by Kevin Harding, Peisen S. Huang, Toru Yoshizawa, Proceedings of SPIE Vol. 7855 (SPIE, Bellingham, WA, 2010) Article CID Number.

ISSN 0277-786X

ISBN 9780819483850

Published by

SPIE

P.O. Box 10, Bellingham, Washington $98227-0010$ USA

Telephone +1 3606763290 (Pacific Time) · Fax +1 3606471445

SPIE.org

Copyright (C) 2010, Society of Photo-Optical Instrumentation Engineers

Copying of material in this book for internal or personal use, or for the internal or personal use of specific clients, beyond the fair use provisions granted by the U.S. Copyright Law is authorized by SPIE subject to payment of copying fees. The Transactional Reporting Service base fee for this volume is $\$ 18.00$ per article (or portion thereof), which should be paid directly to the Copyright Clearance Center (CCC), 222 Rosewood Drive, Danvers, MA 01923. Payment may also be made electronically through CCC Online at copyright.com. Other copying for republication, resale, advertising or promotion, or any form of systematic or multiple reproduction of any material in this book is prohibited except with permission in writing from the publisher. The CCC fee code is 0277-786X/10/\$18.00.

Printed in the United States of America.

Publication of record for individual papers is online in the SPIE Digital Library.

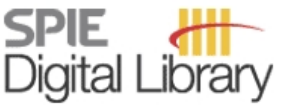

SPIEDigitalLibrary.org

Paper Numbering: Proceedings of SPIE follow an e-First publication model, with papers published first online and then in print and on CD-ROM. Papers are published as they are submitted and meet publication criteria. A unique, consistent, permanent citation identifier (CID) number is assigned to each article at the time of the first publication. Utilization of CIDs allows articles to be fully citable as soon they are published online, and connects the same identifier to all online, print, and electronic versions of the publication. SPIE uses a six-digit CID article numbering system in which:

- The first four digits correspond to the SPIE volume number.

- The last two digits indicate publication order within the volume using a Base 36 numbering system employing both numerals and letters. These two-number sets start with 00, 01, 02, 03, 04 , 05, 06, 07, 08, 09, OA, OB ... 0Z, followed by 10-1Z, 20-2Z, etc.

The CID number appears on each page of the manuscript. The complete citation is used on the first page, and an abbreviated version on subsequent pages. Numbers in the index correspond to the last two digits of the six-digit CID number. 


\title{
Contents
}

\author{
ix Symposium Committees \\ xi Conference Committee \\ xiii Fibre optic systems for gas detection principles, progress, and prospects (Plenary Paper) \\ [7853-150] \\ B. Culshaw, Univ. of Strathclyde (United Kingdom)
}

\section{SESSION 1 OPTICAL METROLOGY FOR NONDESTRUCTIVE TESTING}

785502 PEM-based polarimeters for industrial applications (Invited Paper) [7855-01]

B. Wang, Hinds Instruments, Inc. (United States)

785503 Computer simulation of photomechanics [7855-02]

D. Yan, S. Ma, Beijing Institute of Technology (China)

785504 Spectroscopic topological Stokes polarimeter [7855-03]

T. Wakayama, Saitama Medical Univ. (Japan); Y. Otani, Utsunomiya Univ. (Japan);

T. Yoshizawa, Saitama Medical Univ. (Japan)

785507 Measurement of the elastic modulus of solid material with objective speckles field [7855-06] P. Ran, Z. Fan, H. Xia, Kunming Univ. of Science and Technology (China)

785508 Determination of the optimal marker positions for optical extensometer considering lens distortion [7855-07]

Z. Zhao, X. Wang, J. Pang, S. Ma, Beijing Institute of Technology (China)

785509 Error analysis of strain measurement induced by operating temperature of uncooled CCD [7855-08]

J. Pang, Q. Ma, S. Ma, Beijing Institute of Technology (China); H. Wang, Tsinghua Univ. (China)

7855 OA Optical system design for crack inspections using magneto-optical imaging [7855-09]

Q. HU, R. Dougherty, P. Bondurant, QUEST Integrated, Inc. (United States)

\section{SESSION 2 OPTICAL METROLOGY DEVICES}

7855 OB Development of an inner profile measurement instrument using a ring beam device (Invited Paper) [7855-10]

T. Yoshizawa, Saitama Medical Univ. (Japan) and 3D Associates (Japan); T. Wakayama, Saitama Medical Univ. (Japan)

7855 OD A palm-top camera for 3D profilometry incorporating a MEMS scanner [7855-12]

T. Yoshizawa, T. Wakayama, Saitama Medical Univ. (Japan) 
7855 OE Phase shift based measurements using a pocket LCD projector [7855-13] Y. Williams, K. Harding, GE Global Research (United States)

$7855 \mathrm{OF} \quad$ A hand-held triangulation sensor for small features measurement [7855-14] G. Abramovich, K. Harding, GE Global Research (United States)

7855 OG A simple optical system for measuring small rotation angle of mechanism [7855-15] W. Li, Institute of Electrical Engineering (China); Q. Ma, Beijing Institute of Technology (China); D. Li, S. Liu, C. Yuan, Institute of Electrical Engineering (China)

$7855 \mathrm{OH}$ Two-coordinate dynamic photoelectric autocollimator based on single linear CCD [7855-16]

Z. Bian, M. Gao, Z. Dong, Q. Ye, R. Qu, Z. Fang, Shanghai Institute of Optics and Fine Mechanics (China)

78550 Ol Absolute phase calculation from one composite RGB fringe pattern image by windowed Fourier transform algorithm [7855-17]

Z. Zhang, Z. Jing, H. Feng, H. Ma, Hebei Univ. of Technology (China); T. Guo, Tianjin Univ. (China); S. Zhang, Hebei Univ. of Technology (China)

7855 0J High-resolution dynamic three-dimensional profilomety based on a combination of stereovision and color-encoded digital fringe projection [7855-18]

D. Li, J. Tian, Shenzhen Univ. (China)

\section{SESSION 3 OPTICAL METROLOGY METHODS}

7855 OK Profilometry using Fizeau-interferometer based on optical comb interferometry and sinusoidal phase modulation method [7855-19]

S. Choi, H. Miyatsuka, O. Sasaki, T. Suzuki, Niigata Univ. (Japan)

$7855 \mathrm{OL} \quad$ A displacement reconstruction algorithm used for optical feedback self-mixing interferometry system under different feedback levels [7855-20]

Y. Fan, Y. YU, J. Xi, J. F. Chicharo, Univ. of Wollongong (Australia); H. Ye, Zhengzhou Univ. (China)

7855 OM FPGA-based signal processing in an optical feedback self-mixing interferometry system [7855-21]

Z. Li, Y. YU, J. Xi, Univ. of Wollongong (Australia); H. Ye, Zhengzhou Univ. (China)

7855 ON Time-resolved vibrational surface profile measurement of ultrasonic motor using stroboscopic oblique incidence interferometer [7855-22]

Y. Mizutani, T. Iwata, Univ. of Tokushima (Japan); Y. Otani, Utsunomiya Univ. (Japan)

7855 OP Theoretical analysis of the frequency splitting caused by intracavity quartz crystal [7855-24]

Z. Hu, Beijing Univ. (China); S. Zhang, Tsinghua Univ. (China)

$78550 Q \quad$ Optical FMCW interference: a new technology for optical metrology [7855-25]

G. Zheng, Beijing Institute of Technology (China) 
7855 OR Profile measurement based on spectral interferometer with multi-wavelength back-propagation methods [7855-26]

K. Otsuki, S. Choi, O. Sasaki, T. Suzuki, Niigata Univ. (Japan)

7855 OS Sinusoidal wavelength-scanning common-path interferometer with a beam-scanning system for measurement of film thickness variations [7855-27]

O. Sasaki, T. Morimatsu, S. Choi, T. Suzuki, Niigata Univ. (Japan)

\section{SESSION 4 ANALYSIS AND CALIBRATION METHODS FOR OPTICAL METROLOGY}

7855 OT Error analysis for 3D shape measurement with projector defocusing [7855-28]

Y. Xu, lowa State Univ. (United States); J. Dai, Zhejiang Univ. (United States); S. Zhang, lowa State Univ. (United States)

7855 OU Calibration of a phase-based 3D imaging system based on uneven fringe projection technique [7855-29]

Z. Zhang, H. Ma, H. Feng, Z. Jing, Hebei Univ. of Technology (China); T. Guo, Tianjin Univ. (China); S. Zhang, Hebei Univ. of Technology (China)

7855 OV Calibration target reconstruction for 3-D vision inspection system of large-scale engineering objects [7855-30]

Y. Yin, Tianjin Univ. (China); X. Peng, Tianjin Univ. (China) and Shenzhen Univ. (China);

Y. Guan, Tianjin Univ. (China); X. Liu, A. Li, Shenzhen Univ. (China)

7855 OW Phase error correction based on Inverse Function Shift Estimation in Phase Shifting Profilometry using a digital video projector [7855-31]

Y. Liu, J. Xi, Y. YU, J. Chicharo, Univ. of Wollongong (Australia)

7855 OX Fast quality-guided flood-fill phase unwrapping algorithm for three-dimensional fringe pattern profilometry [7855-32]

K. Chen, J. Xi, Y. Yu, J. F. Chicharo, Univ. of Wollongong (Australia)

7855 OY Calibration and image enhancement algorithm of portable structured light 3D gauge system for improving accuracy [7855-33]

L. Tao, GE Global Research (China); K. Harding, GE Global Research (United States); M. Jia,

G. Song, GE Global Research (China)

$7855 \mathrm{OZ} \quad$ Measuring method for the object pose based on monocular vision technology [7855-34]

C. Sun, Z. Zhang, P. Wang, Tianjin Univ. (China)

785510 Effect of color illumination on color contrast in color vision application [7855-35]

Z. Zhu, X. QU, H. Liang, G. Jia, Tianjin Univ. (China)

785511 An anti-noise subpixel algorithm based on phase-shifting of Fourier transform and its application in CCD photoelectric autocollimator [7855-36]

M. Gao, Z. Bian, Shanghai Institute of Optics and Fine Mechanics (China) and Graduate Univ. of Chinese Academy of Sciences (China); Z. Dong, Q. Ye, Z. Fang, R. Qu, Shanghai Institute of Optics and Fine Mechanics (China) 
785512 Effect of structural parameters on the performance of fiber distance sensor with single mode illumination and inclined-fiber receiving [7855-37]

Z. Zhong, H. Peng, M. Shan, L. Guo, Harbin Engineering Univ. (China)

\section{SESSION 5 OPTICAL METROLOGY APPLICATIONS}

785513 3D profilometry: next requests from the industrial viewpoint (Invited Paper) [7855-38]

K. Harding, GE Global Research (United States)

785514 Optical coherence tomography used for jade industry [7855-39]

S. Chang, Y. Mao, National Research Council Canada (Canada); G. Chang, Shandong

Yingcai Univ. (China); C. Flueraru, National Research Council Canada (Canada)

785515 A fast three-dimensional reconstruction method applied for the fabric defect detection [7855-40]

L. Song, C. Zhang, H. Xiong, Y. Wei, H. Chen, Tianjin Polytechnic Univ. (China)

785516 A novel method to measure wheelset parameters based on laser displacement sensor on line [7855-41]

Z. Zhang, Y. Su, Zhengzhou Univ. of Light Industry (China); Z. Gao, Beijing Jiaotong Univ. (China); G. Wang, Northwestern Polytechnical Univ. (China); Y. Ren, F. Jiang, Zhengzhou Univ. of Light Industry (China)

785517 Steam wetness measurement using CCD imaging methods in low-pressure turbine [7855-42] W. Wu, S. Qin, National Univ. of Defense Technology (China); Z. Huang, Changsha Univ. of Science and Technology (China); X. Wang, C. Hu, National Univ. of Defense Technology (China)

785519 Green inspection station [7855-45]

C.-K. Sung, A. Jacubasch, Fraunhofer-Institut für Optronik, Systemtechnik und

Bildauswertung (Germany)

\section{POSTER SESSION}

$78551 \mathrm{C}$ The influence of BRDF calibration to CT industry NDT [7855-47]

Z. Liu, Beijing Institute of Technology (China) and National Institute of Metrology (China);

N. Liao, Beijing Institute of Technology (China)

7855 1D The study of interferometer spectrometer based on DSP and linear CCD [7855-48]

H. Kang, Y. Peng, X. XU, X. Xing, Beijing Univ. of Technology (China)

$7855 \mathrm{IE}$ Study on a novel illuminance calibration method for signal to noise ratio measurement of image intensifier [7855-49]

J. Shi, H. Li, D. Cui, F. Cao, Y. Sun, Q. Xie, Xi'an Institute of Applied Optics (China)

$7855 \mathrm{IF}$ 3D profile measurement by using projection speckle pattern correlation method [7855-50] E. Hu, L. Zhu, China Univ. of Mining and Technology (China)

7855 1G Design of 3D vision probe based on auto-focus [7855-51]

Q. Liu, D. Yuan, B. Liu, China Academy of Engineering Physics (China) 
$78551 \mathrm{H} \quad$ Principle of a novel displacement sensor based on infrared He-Ne laser [7855-52] Z. Zhao, S. Zhang, Y. Li, Tsinghua Univ. (China)

$785511 \quad$ Pose estimation from four corresponding points with a single camera [7855-53] P. Wang, Y. Zhou, Q. Zhang, Science and Technology on Electro-optic Control Lab. (China)

$78551 \mathrm{~J} \quad$ Ultraviolet bidirectional reflectance distribution function measurement and analysis of typical roughness surface [7855-54]

L. Bai, H. Zhang, Y. Cao, H. Li, Z. Wu, Xidian Univ. (China); S. Wang, Anhui Institute of Optics and Fine Mechanics (China)

$78551 \mathrm{~K}$ Application of photoelectric autocollimator in detecting position precision of NC motorized stage [7855-55]

B. Yan, Beijing Information Science and Technology Univ. (China); Q. Tan, Beijing Univ. of Posts and Telecommunications (China); N. Lv, Beijing Information Science and Technology Univ. (China)

$78551 \mathrm{~L}$ Distance measurement for curved surface based on confocal technique [7855-56] J. Luo, Zhejiang Univ. (China) and Zhejiang Sci-Tech Univ. (China); Y. Liang, Zhejiang Univ. (China); W. Ding, Hangzhou Special Equipment Inspection Institute (China); G. Yang, Zhejiang Univ. (China)

$78551 \mathrm{M} \quad$ Fluorescence rejection by shifted excitation Raman difference spectroscopy [7855-57] W. Zou, Z. Cai, J. Wu, Soochow Univ. (China)

785510 Computer simulation of phase-shift algorithm [7855-59]

G. Wang, J. Yan, Beijing Institute of Technology (China)

$78551 Q \quad$ A real-time multipoint tracking system based on FPGA for multi-touch and motion tracking [7855-61]

X. Hu, Q. Li, X. Li, Beijing Institute of Technology (China)

7855 1T The research of on-line inspection method of printed matter based on optical information processing [7855-64]

J. Wang, Beijing Institute of Graphic Communication (China)

$78551 \mathrm{U} \quad$ A laser self-mixing interference vibrometer based on current modulation and DSP demodulation [7855-65]

W. Xia, M. Wang, W. Guo, Nanjing Normal Univ. (China)

$78551 \mathrm{~W}$ The development of optical fringe measurement system integrated with a CMM for products inspection [7855-67]

H. Xiong, M. Pan, X. Zhang, Guangdong Univ. of Technology (China)

$78551 Z$ Influence of surface material characteristics on laser radar 3D imaging of targets [7855-70] B. Wang, Z.-S. Wu, Y.-J. Gong, Xidian Univ. (China)

785521 CCD calibration method for wheel set wear online measurement [7855-72]

J. Chen, K. Wu, Hangzhou Dianzi Univ. (China) 
785522 Three registration strategies for point clouds in optical inspection of free form shaped plastic parts [7855-73]

H. Xiong, M. Pan, X. Zhang, Guangdong Univ. of Technology (China)

785524 Algorithm for determining line centre with microscope measuring system [7855-75]

H. Wei, W. Wang, G. Ren, L. Pei, National Institute of Metrology (China)

785525 An in-situ monitoring system on the grinding process [7855-76]

B. Kim, M. Kwon, Kyungnam Univ. (Korea, Republic of); J. Ha, K. Lee, ChangWon Precision Ind. Co., Ltd. (Korea, Republic of)

785526 Automatic 3D point clouds registration method [7855-77]

T. Wu, N. Lv, X. Lou, P. Sun, Beijing Information Science and Technology Univ. (China)

785527 Research on performance evaluation of non-arm coordinate measuring machine (NACMM) [7855-78]

W. Wang, H. Wei, National Institute of Metrology (China); Y. Xia, Beijing Institute of Metrology (China)

785529 Position and orientation measurement for large-size workpiece based on binocular vision [7855-80]

F. Liu, X. Lou, N. Lv, P. Sun, Beijing Information Science and Technology Univ. (China)

7855 2A UV light source adaptive sensing technology for flue gas measurement [7855-81]

C. Sun, C. Zhang, B. Sun, B. Liu, P. Wang, Tianjin Univ. (China)

7855 2B Design and implementation of automatic opto-electrical detection system for spheroidal graphite cast iron metallographic phase [7855-82]

Q. Meng, Z. Xiao, S. Deng, Guilin Univ. of Electronic Technology (China)

$78552 \mathrm{C}$ The influence of flame fusion synthetic ruby red on color difference [7855-83]

X. Li, Y. Guo, China Univ. of Geosciences (China)

Author Index 


\title{
Symposium Committees
}

\author{
General Chairs
}

Ralph James, Brookhaven National Laboratory (United States)

Bingkun Zhou, Tsinghua University (China)

General Cochairs

Arthur Chiou, National Yang-Ming University (Taiwan, China)

Zhizhan Xu, Shanghai Institute of Optics and Fine Mechanics (China)

Jianlin Cao, China Ministry of Science and Technology (China)

Junhao Chu, Shanghai Institute of Technical Physics (China)

Honorary Chairs

Daheng Wang, Chinese Academy of Sciences (China)

Guoguang Mu, Nankai University (China)

Technical Program Chair

Songlin Zhuang, Shanghai University of Science and Technology (China)

Technical Program Cochairs

Qiming Wang, Institute of Semiconductors (China)

Xu Liu, Zhejiang University (China)

Qihuang Gong, Peking University (China)

Yimo Zhang, Tianjin University (China)

Local Organizing Committee Chair

Shusen Xie, Fujian Normal University (China)

Local Organizing Committee Cochairs

Guoqiang Ni, Beijing Institute of Technology (China)

Xiaomin Ren, Beijing University of Posts and Telecommunications (China)

Daoyin Yu, Tianjin University (China)

Ying Gu, PLA General Hospital (China)

Huilin Jiang, Changchun University of Science and Technology (China) 
General Secretary

Guoqiang Ni, Beijing Institute of Technology (China)

Administrative Vice General Secretary

Boyu Ding, Beijing Institute of Technology (China)

Vice General Secretaries

Hanyi Zhang, Tsinghua University (China)

Ya Cheng, Shanghai Institute of Optics and Fine Mechanics (China)

Zhongwei Fan, Academy of Opto-electronics (China)

Jianxin Chen, Fujian Normal University (China)

Lan Wu, Zhejiang University (China)

Jianping Zhang, Chinese Optical Society (China)

Local Organizing Committee

Chongxiu Yu, Beijing University of Posts and Telecommunications

(China)

Hongda Chen, Institute of Semiconductors (China)

Yuejin Zhao, Beijing Institute of Technology (China)

Kangnan Qi, Beijing Optical Society (China)

Chunqing Gao, Beijing Institute of Technology (China)

Tiegen Liu, Tianjin University (China)

Xiaocong Yuan, Nankai University (China)

Weiming Chen, Chongqing University (China)

Lanxing Shao, Shanghai Optical Society (China)

Baosheng Liu, Beijing Optical Society (China)

Xin Wang, Hubei Optical Society (China)

Lin Zhai, Chinese Optical Society (China) 


\title{
Conference Committee
}

\author{
Conference Chairs \\ Kevin Harding, GE Global Research (United States) \\ Peisen S. Huang, Stony Brook University (United States) \\ Toru Yoshizawa, Saitama Medical University (Japan)
}

Program Committee

Yasuhiko Arai, Kansai University (Japan)

Qingying Hu, Akrometrix LLC (United States)

Lianhua Jin, University of Yamanashi (Japan)

Jun-ichi Kato, RIKEN (Japan)

Katsuichi Kitagawa, Toray Precision Company, Ltd. (Japan)

Peter Kuehmstedt, Fraunhofer-Institut für Angewandte Optik und Feinmechanik (Germany)

Guangrong Liu, Beijing Institute of Technology (China)

Yumi Mori, IBM Japan, Ltd. (Japan)

Takashi Nomura, Toyama Prefectural University (Japan)

Yukitoshi Otani, Tokyo University of Agriculture and Technology (Japan)

Guiju Song, GE Global Research (China)

Joseph D. Tobiason, Micro Encoder Inc. (United States)

Rainer Tutsch, Technische Universität Braunschweig (Germany)

Jiangtao Xi, University of Wollongong (Australia)

Jian Xu, A*STAR Singapore Institute of Manufacturing Technology

(Singapore)

Hao Zhang, Tianjin University (China)

Song Zhang, lowa State University (United States)

Session Chairs

1 Optical Metrology for Nondestructive Testing

Toru Yoshizawa, Saitama Medical University (Japan)

2 Optical Metrology Devices

Kevin Harding, GE Global Research (United States)

3 Optical Metrology Methods

Peisen S. Huang, Stony Brook University (United States)

Guiju Song, GE Global Research (China) 
$4 \quad$ Analysis and Calibration Methods for Optical Metrology Qingying Hu, QUEST Integrated, Inc. (United States)

5 Optical Metrology Applications

Jiangtao Xi, University of Wollongong (Australia) 